\title{
Protocol for a collaborative meta-analysis of 5-HTTLPR, stress, and depression
}

Robert C Culverhouse ${ }^{1,2^{*}}$, Lucy Bowes ${ }^{3}$, Naomi Breslau4 ${ }^{4}$, John I Nurnberger $\mathrm{Jr}^{5}$, Margit Burmeister ${ }^{6,7,8}$, David M Fergusson ${ }^{9}$, Marcus Munafò ${ }^{10}$, Nancy L Saccone ${ }^{11}$, Laura J Bierut ${ }^{12}$ and On behalf of the 5-HTTLPR, Stress, and Depression Consortium

\begin{abstract}
Background: Debate is ongoing about what role, if any, variation in the serotonin transporter linked polymorphic region (5-HTTLPR) plays in depression. Some studies report an interaction between 5-HTTLPR variation and stressful life events affecting the risk for depression, others report a main effect of 5-HTTLPR variation on depression, while others find no evidence for either a main or interaction effect. Meta-analyses of multiple studies have also reached differing conclusions.
\end{abstract}

Methods/Design: To improve understanding of the combined roles of 5-HTTLPR variation and stress in the development of depression, we are conducting a meta-analysis of multiple independent datasets. This coordinated approach utilizes new analyses performed with centrally-developed, standardized scripts. This publication documents the protocol for this collaborative, consortium-based meta-analysis of 5-HTTLPR variation, stress, and depression.

Study eligibility criteria: Our goal is to invite all datasets, published or unpublished, with 5-HTTLPR genotype and assessments of stress and depression for at least 300 subjects. This inclusive approach is to minimize potential impact from publication bias.

Data sources: This project currently includes investigators from 35 independent groups, providing data on at least $\mathrm{N}=33,761$ participants.

The analytic plan was determined prior to starting data analysis. Analyses of individual study datasets will be performed by the investigators who collected the data using centrally-developed standardized analysis scripts to ensure a consistent analytical approach across sites. The consortium as a group will review and interpret the meta-analysis results.

Discussion: Variation in 5-HTTLPR is hypothesized to moderate the response to stress on depression. To test specific hypotheses about the role of 5-HTTLPR variation on depression, we will perform coordinated meta-analyses of de novo results obtained from all available data, using variables and analyses determined a priori. Primary analyses, based on the original 2003 report by Caspi and colleagues of a GxE interaction will be supplemented by secondary analyses to help interpret and clarify issues ranging from the mechanism of effect to heterogeneity among the contributing studies. Publication of this protocol serves to protect this project from biased reporting and to improve the ability of readers to interpret the results of this specific meta-analysis upon its completion.

\footnotetext{
* Correspondence: rculverh@wustl.edu

${ }^{1}$ Department of Medicine, Washington University School of Medicine, St.

Louis, MO, USA

${ }^{2}$ Division of Biostatistics, Washington University School of Medicine, St. Louis, $\mathrm{MO}$, USA

Full list of author information is available at the end of the article
}

\section{Biomed Central}

(C) 2013 Culverhouse et al.; licensee BioMed Central Ltd. This is an Open Access article distributed under the terms of the Creative Commons Attribution License (http://creativecommons.org/licenses/by/2.0), which permits unrestricted use, distribution, and reproduction in any medium, provided the original work is properly cited. The Creative Commons Public Domain Dedication waiver (http://creativecommons.org/publicdomain/zero/1.0/) applies to the data made available in this article, unless otherwise stated. 


\section{Introduction}

Large-scale, collaborative meta-analysis has the potential to clarify complex scientific questions by increasing sample size, harmonizing variables, and conducting uniform analyses. In this paper, we describe the protocol for a collaborative, consortium-based meta-analysis to increase understanding of the role of 5-HTTLPR variation and stress in depression. The publication of this protocol serves several important purposes. First, we document our study protocols, design, and primary analysis decisions prior to conducting and publishing our study of 5HTTLPR variation, stress, and depression, which will avoid biased reporting later. Second, publication of these details will improve the ability of other researchers to interpret the results of this specific meta-analysis upon its completion. Finally, publishing our detailed protocol may be helpful to future consortia by detailing key analysis strategies and as a standard for transparency and commitment to analytic plans set prior to meta-analysis.

\section{Background}

Both genetic and environmental factors influence depression [1]. As with other psychiatric illnesses, research on the etiology of depression has identified moderate heritability estimates of 40-50\% [1-6], yet recent genome-wide association studies (GWAS) have so far been unable to identify robust and replicable loci associated with depression [7]. It has been argued that some of this 'missing heritability' is a result of particular genes exerting an influence on risk for depression only under specific environmental conditions, or 'gene-environment interactions $(\mathrm{GxE})[8,9]$. One of the most high profile reports of GxE involves a common functional polymorphism (5-HTTLPR) in the promoter region of the serotonin transporter gene (5-HTT). This gene encodes an integral membrane protein that transports the neurotransmitter serotonin from synaptic spaces into presynaptic neurons, which terminates the action of serotonin. The repeat length polymorphism has been shown to affect the rate of serotonin uptake [10]. Specifically, the short (S) allele of the 5-HTTLPR is associated with less transcriptional efficiency of the promoter compared to the long (L) allele [11]. Additionally, a single nucleotide substitution (rs25531, A > G) within both alleles reduces transcription so that the $\mathrm{L}_{\mathrm{G}}$ allele becomes functionally equivalent to the $\mathrm{S}$ allele $[12,13]$. Studies have suggested grouping $\mathrm{L}_{\mathrm{G}}$ with the $\mathrm{S}$ allele to increase efficiency in predicting variation in serotonin transporter expression [14], although not all agree on this point.

In 2003, Caspi and colleagues reported evidence of a $\mathrm{G} \times \mathrm{E}$ interaction between 5 -HTTLPR variation and stressful life events on depression [15], with nearly 5000 citations to date. Carriers of either one or two copies of the $S$ allele of the 5-HTTLPR were reported to be more likely to develop major depressive disorder, increased depressive symptoms, and suicidality in response to stressful life events and, separately, child maltreatment than individuals homozygous for the L allele. Furthermore, there was evidence of a dose-response relationship, with risk of depression highest amongst those with two copies of the $S$ allele compared to individuals with only one copy in the presence of stress.

In the original report, no main effect of genotype was found. If the genotype exerts an effect only on those exposed to the stressor, i.e. a diathesis-stress model, the lack of main effect may be due to insufficient power [16]. Alternatively, the genotypic effect may be one of differential environmental susceptibility [17], in which the L-carriers are indifferent to the environment, whereas the $\mathrm{S}$ allele confers environmental susceptibility, allowing $\mathrm{S}$ carriers to benefit more from positive experiences as well as being more sensitive to stress, resulting in no net genotype-depression association irrespective of sample size [18]. Studying a large sample may distinguish these possibilities.

Since the original report of a GxE interaction, hundreds of studies have investigated the combined impact of 5-HTTLPR variation and stress on risk for depression, some of which reported replicating the original findings, while some did not. Meta-analyses, also, have come to quite different conclusions [16,19-22] and various reasons for these differences have been proposed [21,23-25].

Below we discuss 4 key factors that complicate the interpretation of existing results related to the interplay between 5-HTTLPR variation, stress and depression.

1. Study design. One factor complicating interpretation is differences in study design. Sample sizes vary, with the majority modest or small. Underpowered studies, combined with potential publication bias, can lead to an increased risk of Type 1 errors [26]. Sampling strategies vary from population-based methods $[15,27]$ to convenience sampling $[28,29]$. Different ancestral populations have been included, with a preponderance of samples of European ancestry. The age range of subjects varies, and it has been suggested that GxE effects are most consistently replicated in young adult samples [18].

The studies contributing to our meta-analysis represent a range of study designs, so we will test whether differing study design contributes to the heterogeneity of results.

2. Timing of measurements. Brown and Harris [19] noted that studies that have failed to replicate the GxE between 5-HTTLPR variation and life events have measured the occurrence of stressful life events in the months immediately preceding the depressive outcomes [19]. In contrast, most of the positive replications have been in keeping with the original study 
by Caspi and colleagues in measuring life events in the five years prior to the outcome. Retrospective recall of adversity over long periods of time may increase the risk of forgetting or discounting of events [30] or lead to a bias due to selective recall when those who are depressed self report $[27,31]$. When only a lifetime diagnosis of depression is available, information about relative timing of stressors and depression is lost.

Longitudinal studies are largely able to avoid this bias, so our test of longitudinal vs. cross-sectional designs will also, in part, address the issue of timing of stress and depression.

3. Type of environmental stressor. Different stressors have been examined for interaction with 5-HTTLPR variation. The most common are broad measures of stressful life events $[32,33]$ and exposure to child maltreatment $[34,35]$. The method of measurement (self report vs. interviewer), the type of stressors (e.g. chronic vs. acute) and the scale (binary exposed vs. not exposed, frequency as in the original study, or continuous variable) also vary. It has been suggested that the varied methodology in assessing stressful life events in GxE studies may in part explain the inconsistency of findings [18], and in a meta-analysis that differentiated between stressors (child maltreatment, specific stressors, and stressful life events), significant differences between types of stressors were found.

We will therefore perform heterogeneity analyses to account for different types of stressors and measurements of stressors. One such test will be to compare results from the studies that focused on specific stressors (e.g. pregnancy, heart attack, medical internship) to those from studies based on summary measures of diverse stressors (e.g. the LTE-Q).

4. Type of outcome. Some studies used a categorical measure of depression diagnosis (e.g. DSM-IV or the ICD-10) as an outcome, others used a symptom count as continuous outcome, and some used both. Such differences in outcome measures (e.g., continuous versus categorical) result in different assumptions and analytical approaches being used (see for a Discussion [36]).

The role of 5-HTTLPR variation and stress in the development of depression remains a topic of active debate, in part because of the challenges outlined above. Therefore, we are undertaking this collaborative meta-analysis using a standardized protocol to improve understanding of this important issue.

\section{Study objectives}

The primary objective of this study is to increase understanding of the role of 5-HTTLPR variation and stress in depression, where 5-HTTLPR variation is hypothesized as a moderator of the response to stress. To address the complexities of this topic, we will perform a coordinated meta-analysis of all data available from collaborators, using consistent de novo analyses and variables determined a priori.

In keeping with the original finding by Caspi and colleagues [15], we will test the following main hypothesis.

The risk of depression, evaluated either as a dichotomous diagnosis or as a continuous phenotype, is greater in carriers of the $\mathrm{S}$ allele versus those homozygous for the $\mathrm{L}$ allele in the presence of exposure to stress.

This main hypothesis will be examined in multiple settings to determine a range of conditions under which the effect might be found. Additional hypotheses (e.g. whether there is a main effect of 5-HTTLPR variation on depression) will be examined to improve our understanding of this complex topic.

\section{Methods/Design \\ Overview}

Plans for analysis, definitions of stressors and outcomes, as laid out in this protocol, were determined a priori by our study leadership team in consultation with contributing study investigators, who provided input via conference calls and email. Individual study analyses will be performed by the investigators who collected the data, using standardized analysis scripts developed by the coordinating team at Washington University to implement a consistent analytical approach across sites. Results from these analyses will be sent to the coordinating team for meta-analysis. The consortium as a group will review and interpret the results. We have previously developed and successfully implemented this collaborative meta-analysis approach to investigate the genetics of addiction [37-39].

This coordinated meta-analysis approach has several advantages over traditional literature-based meta-analyses arising from the fact that a collaborative meta-analysis is based on de novo, consistent analyses of harmonized variables in the primary data. First, the consistent analytic approach and harmonized variables greatly ameliorate the effects of two of the chief causes of heterogeneity between individual studies. In particular, meta-analyzing results derived from differing analytic approaches, such as the use of different covariates, different phenotype definitions, and different analytical models, has a clear potential to bias literature-based meta-analyses [40]. Second, the fact that the results are based on de novo analyses allows for the inclusion of previously unpublished data, both from researchers who have not published on the topic before and through updated individual data from research groups who have previously published. Third, coordinated de novo analyses of primary data allow a collaborative metaanalysis to conduct secondary analyses that can aid in the 
interpretation of the main results. Finally, the coordinated meta-analysis approach allows the examination of more homogeneous subsets of the primary data than is possible through literature review.

\section{Identifying studies}

Our goal is to invite participation from all studies with datasets that have the 5-HTTLPR variant genotyped, and assessments of stress and depression, on at least 300 subjects. We set a minimum study size anticipating that the individuals available for our de novo analyses would typically be only a subset of the study individuals. Setting an arbitrary study minimum was intended to improve the balance between the heterogeneity introduced by a new study and the increased sample size. It also had the potential to ameliorate bias due to a possible publication bias amongst small studies. One drawback to this approach of requiring large sample size is that it may exclude some studies with the most intensive assessments of stress and depression. Invitations were sent to groups that had previously published on the topic. Additional invitations were sent to groups, published or unpublished on this topic, suggested as a potentially qualifying study by a participating group. The goal of this inclusive approach is to minimize the potential impact of publication bias. The participating collaborative studies are listed in Table 1. All participating studies have approval from their local boards governing ethical conduct of research in human subjects to conduct the agreed upon statistical analyses. A list of the review boards can be found in the acknowledgements. Additional groups that have been invited, but for a variety of reasons are not currently participating, are identified by publications listed in Additional file 1: Table S1.

Inclusion criteria:

- 5-HTTLPR variant genotyped.

- Measurement of exposure to stress (including stressful life events and/or maltreatment).

- Measurement of depression (including symptom counts and/or diagnosis).

- Study sample size of 300 or more.

Exclusion criteria:

- Studies where the 5-HTTLPR variant was not directly genotyped.

- Studies with a sample size of less than 300 participants.

\section{Data management, analysis, and meta-analysis}

Multiple existing, independent datasets will contribute de novo analysis results for meta-analysis, following coordinated meta-analysis protocols we have developed and implemented previously [37-39]. Extensive meetings between the leaders of the contributing studies have focused on effective ways to harmonize the available variables across the studies (see "Data Harmonization" below) to minimize heterogeneity across the resulting analyses. Following the common protocol, each contributing group will format their data for analysis by a common analysis script. Analyses will be performed by each individual data contributing site, using standardized scripts developed by the coordinating site at Washington University in consultation with the entire consortium. Beta-testing of these scripts will be performed both by the coordinating team and individual study teams. These scripts will ensure consistent analyses across sites. Results will be returned to the coordinating site for meta-analysis. The results of the meta-analyses will be interpreted by the consortium and a collaborative publication reporting the results will be produced.

\section{Quality control}

The data contributing sites will be responsible for checking the quality of their formatted data (e.g., making sure that values for all variables are credible, confirming the sample size, and checking the number of missing observations for each variable) prior to analysis and in the results returned to the coordinating site. The coordinating site will review the results and work out any anomalies with the data providers, and perform meta-analyses.

\section{Data harmonization}

Differences in assessment of stress and depression and information regarding the timing of stress and depression, is a major challenge for effectively combining the information from the participating studies. The process of data harmonization began with surveys of the contributing studies to determine what key variables were available in each dataset. Based on this information, the consortium judiciously balanced the effort of trying to include as many studies as possible against the most appropriate variables for analysis. Below we describe the two key variables and approaches selected for study.

1. Depression: Many different assessments and evaluations are available for study. Differences in measurement of depression may influence the association results, and as a result, a thorough review of the best measurement to be used in this study was undertaken.

The primary phenotype selected for analysis is a major depressive disorder defined by DSM-IV criteria. This phenotype is one used in the original [15] paper. Additional analyses will be completed using DSM-IV major depressive disorder symptom count, again as in keeping with the original study. 
Table 1 Groups participating in the 5-HTTLPR coordinated meta-analysis

\begin{tabular}{|c|c|}
\hline Study & Representatives \\
\hline ALSPAC & Marcus Munafò, Ricardo Araya, Lucy Bowes \\
\hline ASPIS & Nicholas Stefanis, Laura Mandelli, Costas N. Stefanis, Alex Hatzimanolis, Alessandro Serretti \\
\hline ATP & Craig Olsson, Keriann Little \\
\hline BIGSIBS & Alex Todorov, Vesselin Chorbov \\
\hline CHDS & David Fergusson, John Horwood \\
\hline Chinese Academy of Medical Sciences & Xu Qi \\
\hline CoFaMS & Bernhard T. Baune, Grant Sinnamon, Sarah Cohen-Woods \\
\hline COGA & John Nurnberger, Jr. \\
\hline COGEND & Naomi Breslau, Laura Bierut \\
\hline CTS-LTS and NYSFS & John Hewitt, Brett Haberstick \\
\hline DeCC & Peter McGuffin, Helen L. Fisher, Sarah Cohen-Woods, Anne Farmer \\
\hline EPIC-Norfolk & Paul Surtees, Nick Wainwright \\
\hline ESPRIT & Karen Ritchie, Isabelle Jaussent \\
\hline G1219 & Thalia Eley, Kathryn Lester \\
\hline Genetic Study of Bipolar Disorder & Frank Bellivier \\
\hline GENESIS & Philippe Courtet, Alain Malafosse, Emilie Olié \\
\hline GTP & Kerry Ressler, Bekh Bradley-Davino \\
\hline Heart and Soul Study & Christian Otte, Mary Whooley \\
\hline Intern Health Study & Srijan Sen \\
\hline MARS & Manfred Laucht, Tobias Banaschewski, Daniel Brandeis \\
\hline MLS & Margit Burmeister, Robert A Zucker, Sandra Villafuerte \\
\hline Moodlnflame (Münster) & Volker Arolt, Bernhard T. Baune \\
\hline Münster Neuroimaging Study & Udo Dannlowski, Bernhard T. Baune \\
\hline NESDA & Brenda Penninx, Johannes Smit, Wouter Peyrot \\
\hline NEWMOOD & Gabriella Juhasz, Bill Deakin, Gyorgy Bagdy, Judit Lazary \\
\hline NTR (Adult NTR and Young NTR) & Christel Middeldorp, Dorret Boomsma \\
\hline PATH & Simon Easteal, Kaarin J. Anstey \\
\hline $\mathrm{POUCH}$ & Jeanette Scheid, Claudia Holzman, Nicole Jones \\
\hline PREDICT-Gene & Blanca Gutiérrez, Jorge Cervilla, \\
\hline QIMRtwin & Nicholas G. Martin, William L. Coventry, Grant W. Montgomery, Naomi R. Wray \\
\hline SALVe 2001 and SALVe 2006 & Cecilia Åslund, Kent Nilsson \\
\hline SEBAS & Dana Glei, Noreen Goldman, Maxine Weinstein \\
\hline SHIP & Hans Jörgen Grabe \\
\hline TRAILS & Albertine Oldehinkel, Esther Nederhof, Johan Ormel \\
\hline U. Bologna & Laura Mandelli, Alessandro Serretti \\
\hline U. Molise & Marco Sarchiapone, Laura Mandelli \\
\hline VAHCS & George Patton, Craig Olsson, Christina O'Loughlin \\
\hline Coordinating Team & Robert Culverhouse, Nancy Saccone, Laura Bierut, Amy Horton \\
\hline Leadership Team & Robert Culverhouse, Laura Bierut, Naomi Breslau, John Nurnberger Jr., Nancy Saccone \\
\hline External Beta Testers & Lucy Bowes, Sarah Cohen-Woods, Helen Fisher, Brett Haberstick, Marcus Munafò \\
\hline
\end{tabular}

Across the majority of the current contributing datasets, a lifetime diagnosis of DSM-IV major depressive disorder was assessed. For studies where DSM-IV definition of major depressive disorder is not available, a proxy diagnosis will be used. We will track the assessment used and if a proxy diagnostic variable is used, we will examine potential biases introduced by assessment heterogeneity. 
2. Stress: The studies vary widely on the types of assessments of stress and on the relative timing of the stressful event and the onset of depression. This study will examine two main stress assessments: a narrowly focused stressor, childhood maltreatment (most frequently used assessment among our participating groups: the Childhood Trauma Questionnaire (CTQ) [41]), and a broader defined stressor class, lifetime stressful events (most frequently used assessment: the List of Threatening Events (LTE) [42]). A second dimension we will investigate is timing of stress exposure relative to the assessment of depression. Some analyses will require that timing is known, while others will be broadened to include datasets where the timing is uncertain. A comprehensive list of the stress variables used in the analyses can be found in Additional file 2: Table S2. We will test our hypotheses in two models, the first using data from a well-characterized, narrowly defined set of subjects selected to most closely resemble those used in the original study by [15] (young adults with no prior history of depression), and a more broadly defined group of subjects.

\section{Heterogeneity}

Data harmonization requires compromises, and some heterogeneity and biases will surely remain. We will address this issue and improve the interpretation of our primary analyses by testing for heterogeneity across a variety of factors and examining results within and across more homogeneous subsets.

A priori group comparisons for heterogeneity will include the following:

- Longitudinal versus cross-sectional studies.

- Different measures of depression (DSM-IV criteria and other measures).

- Different stress exposure scales.

- Interview versus questionnaire reports.

- Self-report versus report by others.

Should heterogeneity be detected, we will report metaanalysis results from homogeneous subgroups as well as results based on all available data. Assessment of heterogeneity across groups of studies and meta-analyses of sub-groups of studies will be carried out by the coordinating team.

\section{Analysis models}

In our exploration of the relationship between 5-HTTLPR variation, stress, and depression, we will try to determine (1) settings in which an effect can be identified and (2) the extent to which an effect can be generalized.
In the original report by Caspi and colleagues [15], a depression outcome at age 26 was assessed using the Diagnostic Interview Schedule [43], yielding a diagnosis of a major depressive episode according to DSM-IV criteria, as well as a continuous measure of depressive symptoms. Stressful life events occurring over a fiveyear period were assessed retrospectively when study members were aged 26. Subjects with a diagnosis of depression prior to the five-year window were excluded. Stressful events were summed to create a five-level ordinal variable (no life events, one, two, three, and four or more events). Exposure to childhood maltreatment was also examined at three levels (no, probable, and likely maltreatment).

Analyses involved both a logistic regression (multiplicative) model where depression diagnosis was the outcome, and an ordinal least squares regression (additive) model of depressive symptoms. No main effect of genotype was found.

As stated above, our analyses will fall into two main groups of data: a well-characterized, narrowly defined set of subjects similar to those included in the original study, and a broadly defined group of subjects. Each of these groups will be investigated based on two stressor classes: narrow (childhood maltreatment - including physical and sexual abuse as well as physical neglect occurring before age 17) and broad (any lifetime stressful events (e.g. death of family member, job loss, assault, life threatening illness)). The first group of subjects will consist of adults aged 21 to 30, who did not have depression prior to a 5-year window before assessment, and for whom stress exposure is known to have occurred before any current depression. Other than childhood maltreatment, lifetime stressful events will only be considered if they occurred no more than 5 years prior to assessment. The second, broader group of subjects will have no age restriction. Examination of the broader group of subjects will include additional analyses which drop requirements related to the timing of the stressors.

Using these approaches, we intend to balance the competing factors affecting power: sample size and purity of assessments. Analyses of the first class of subjects are designed to most closely match the original report of [15]. By examining the more severe stressor, childhood maltreatment, in a larger sample with no age restriction, our goal is to potentially increase our ability to see the influence of 5-HTTLPR variation if the effect is not limited young adulthood. Our broadest analysis, with stress broadly defined in the largest population, can be expected to maximize power if there is a general effect of 5HTTLPR variation moderating the impact of stress exposure on depression. If we do not see the influence of 5HTTLPR variation in this final set of analyses, but it was present in at least one of the previous analyses, we will be 
able to examine which factors reduce the effect of the association. An important benefit of this design is that we will also examine the degree to which results observed with narrower analyses generalize to the broader analyses, which provides the opportunity to clarify the importance of specific phenotype, stressor, and age requirements.

Under this protocol, data from a particular study may be appropriate for inclusion in one or more of these analysis categories.

\section{Plan details}

The primary model of interest, a focused analysis of 5HTTLPR variation, consists of two arms (arms A and B) and most closely follows the original report. The analysis scripts will also include pre-specified secondary analyses aimed to aid additional understanding of the primary analysis results and to explore secondary hypotheses.

\section{Primary analysis 1: analysis of the impact of 5-HTTLPR variation in young adults}

In keeping with the original study and following reports that effects of any GxE may be expected to be greatest among young adults [18], we will first restrict our analyses to individuals with outcome measures at age 21-30 years.

Two arms differentiated by stressor will be the coprimary foci for this set of analyses.

\section{Arm A: childhood maltreatment}

\section{- Narrower Stressor}

- No time limit on gap between maltreatment and depression

- Stress exposure assumed to occur before depression, but need not be documented

Childhood maltreatment has been reported as a particular stressor of interest for understanding the relationship between 5-HTTLPR variation and depression. The effects of childhood maltreatment were deemed by the consortium members to have long-lasting adverse effects. As a consequence, no time limit between exposure to childhood maltreatment and adult depression outcome will be required. This is also in keeping with the original study. Furthermore, these analyses will assume that childhood maltreatment antedates the development of depression, so specific timing of when the maltreatment occurred relative to any depressive episodes will not be required.

\section{Arm B: stressful life events (including childhood maltreatment)}

- Broader Stressor

- Stressors other than childhood maltreatment must occur $<5$ years prior to assessment
The second arm will take a broader measure of stress, incorporating both stressful life events and maltreatment history. Because stressful life events other than childhood maltreatment were deemed as less likely to have a lifelong impact, for this analysis, only life stressors occurring within 5 years of the outcome measure will be used. Furthermore, in order to elucidate the issue of causation in the event that an association is observed, only data in which the relative timing of exposure to stressful life events and depression outcome is known will be used. Individuals with a history of depression prior to stress exposure will be excluded from these analyses.

\section{Key covariates}

Several covariates may alter the relationship between 5HTTLPR variation, stress, and depression. Effects due to sex, age, birth cohort, and genetic ancestry will be investigated in our analysis.

\section{Sex}

Analyses stratified by sex and using sex as a covariate will be conducted.

\section{Age}

Age at interview will be included as a covariate in the primary analyses.

\section{Birth cohort}

Cohort effects will be examined using decade of birth as a covariate. The primary models will not include birth cohort as a covariate.

\section{Genetic ancestry}

Our analyses will stratified by genetic ancestry (European, Asian, African, Pacific Island, and Admixed EuropeanAfrican). The majority of data is from participants of European ancestry and there may be limited power to detect ethnic heterogeneity in the results. If there is sufficient power to effectively test for heterogeneity and none is found, then a meta-analysis across all ancestry groups will be evaluated.

\section{Statistical models for primary analysis 1 \\ Arm A analysis}

Analysis model 1A1 Moderated logistic regression (dichotomous phenotypes).

$$
\mathrm{D}=\mu+\beta_{1} \mathrm{M}+\beta_{2} \mathrm{G}+\beta_{3}(\mathrm{M} \times \mathrm{G})+\sum_{i=4}^{n} \beta_{i} \operatorname{cov}_{i}
$$

where

$\mathrm{D}=$ diagnosis of DSM-IV major depressive disorder.

$\mathrm{M}=$ dichotomous measure of exposure to childhood maltreatment.

$\mathrm{G}=$ 5-HTTLPR genotype. 
In this analysis, model fit using a likelihood ratio test is compared with and without the interaction term to evaluate whether there is statistically significant evidence for the interaction. Our primary analysis will use an additive coding for genotype based on the number of $\mathrm{S}$ alleles carried and lifetime diagnosis of depression.

Secondary analyses will evaluate whether alternate codings for genotype (such as adding a dominance factor) provide a significantly better fit to the data and the possibility of a main effect of 5-HTTLPR genotype on depression, and investigate both current depression and any depression as alternate outcomes.

Analysis model 1A2 Moderated linear regression (quantitative phenotypes).

$$
\begin{aligned}
\mathrm{D}_{\text {quant }}= & \mu+\beta_{1} \mathrm{M}_{\mathrm{quant}}+\beta_{2} \mathrm{G}+\beta_{3}\left(\mathrm{M}_{\text {quant }} \times \mathrm{G}\right) \\
& +\sum_{\mathrm{i}=4}^{\mathrm{n}} \beta_{\mathrm{i}} \operatorname{cov}_{\mathrm{i}}
\end{aligned}
$$

where

$\mathrm{D}_{\text {quant }}=$ DSM-IV symptom count major depressive disorder.

$\mathrm{M}_{\text {quant }}=$ quantitative measure of exposure to childhood maltreatment.

$\mathrm{G}=$ 5-HTTLPR genotype.

This analysis will parallel the description of Model $1 \mathrm{~A} 1$.

\section{Arm B analysis}

Analysis Models $1 \mathrm{~B} 1$ and $1 \mathrm{~B} 2$ will be analogous to $1 \mathrm{~A} 1$ and $1 \mathrm{~A} 2$, but substituting stressful life events, $\mathrm{S}$, as the stressor. Stressors other than childhood maltreatment must have occurred no more than 5 years prior to the assessment of depression to be included.

These two arms of Primary Analysis 1 will most closely replicate the analyses in the original report. If, as has been suggested, the GxE effect is strongest for childhood maltreatment [19] or is seen primarily in young adults following recent stress exposure [18] this set of analyses will maximize statistical power.

\section{Primary analysis 2: analysis of the impact of 5-HTTLPR variation across all ages}

Our second set of primary analyses will involve larger sample sizes, including children and adults of all ages. The increase in sample size will result in increased power if there is a broad genetic association between 5HTTLPR genotypes, stress, and depression. However, this comes at a cost; in these analyses, we give up the opportunity to investigate whether stress preceding depression was a potential cause of the depression, as relative timing of stress and depression may not be known, and thus will not be included in the models.
Parallel to Primary Analysis 1, these analyses will also utilize two co-primary arms differentiated by stressor.

\section{Arm A: childhood maltreatment}

- Narrower Stressor.

- No time limit on gap between maltreatment and depression.

- Stress exposure assumed to occur before depression, but need not be documented.

As noted above, childhood maltreatment has been reported as a particular stressor of interest for understanding the relationship between 5-HTTLPR variation and depression. This portion of the analysis differs from the first primary analysis Arm A in that there is no limit on the ages of the subjects at the time of interview.

\section{Arm B: stressful life events (including childhood maltreatment)}

- Broader Stressor

- Stressors occurring $<5$ years prior to assessment when information available.

- Stressor timing unknown will not be reason for exclusion.

This arm of the analyses will include a series of nested analyses. The first will be similar to Arm B of the first primary analyses, except that there is no restriction on the ages of the subjects. Additional analyses will further reduce the restrictions on the included data (e.g. timing need not be known) as we attempt to determine what are the broadest general conditions under which an effect can be detected. As we loosen the restrictions, the sample size will increase allowing the possibility of detecting weaker associations in a broader setting.

\section{Statistical models for primary analysis 2}

These analyses will consist of 3 sets of analyses nested based on the subjects that are included.

\section{Analysis models $2 \mathrm{~A} 1 \mathrm{a}$ through $2 \mathrm{~B} 2 \mathrm{a}$}

These are identical to Models 1A1 through 1B2 except that subjects are not restricted to be between age 21 and 30 .

\section{Analysis models $2 \mathrm{~B} 1 \mathrm{~b}$ and $2 \mathrm{~B} 2 \mathrm{~b}$}

These are identical to Models 2A1a through 2B2a except that the timing of the stressful life events relative to depression may be unknown. (Because childhood maltreatment is assumed to occur prior to the first major depressive episode, there are no models $2 \mathrm{~A} 1 \mathrm{~b}$ and 2A2b.) 


\section{Analysis model $2 \mathrm{C}$}

Our broadest analysis will estimate allele frequencies among the cells of a $2 \times 2$ table defined by stress exposure and lifetime diagnosis of major depression, followed by a determination of whether there is evidence of a direct or interaction association between 5-HTTLPR variation and depression.

\section{Secondary models}

As noted above, in addition to the moderated regression models described in the 11 primary analysis models (1A1 through $2 \mathrm{C}$ ), we will examine a variety of secondary models to help us interpret the results of our primary results. Included in these will be analyses where only stresses other than childhood maltreatment are considered. Secondary analyses will also include both alternate statistical models and analyses of more narrowly defined subsets of subjects (e.g. subjects from longitudinal studies, subjects for which the SNP occurring on the $\mathrm{L}$ allele has been genotyped).

Among these secondary analyses will be traditional logistic and linear regression models parallel to the moderated regression models $1 \mathrm{~A} 1$ through $2 \mathrm{~B} 2 \mathrm{~b}$ but without the interaction terms, as illustrated below.

\section{Secondary Models S1A1 \& S1B1}

Traditional logistic regression (dichotomous variables).

$$
\begin{aligned}
& (\mathrm{S} 1 \mathrm{~A} 1) \mathrm{D}=\mu+\beta_{1} \mathrm{M}+\beta_{2} \mathrm{G}+\sum_{\mathrm{i}=3}^{\mathrm{n}} \beta_{\mathrm{i}} \operatorname{cov}_{\mathrm{i}} \\
& \text { and }(\mathrm{S} 1 \mathrm{~B} 1) \mathrm{D}=\mu+\beta_{1} \mathrm{~S}+\beta_{2} \mathrm{G}+\sum_{\mathrm{i}=3}^{\mathrm{n}} \beta_{\mathrm{i}} \operatorname{cov} \nu_{\mathrm{i}}
\end{aligned}
$$

Results from previous studies (e.g. [16]) suggest that there is likely no main effect of 5-HTTLPR variation on depression and as a result, this is not part of our primary analysis. Nonetheless, our secondary analyses will evaluate the possibility of a main effect, both in isolation and as part of larger interaction models (cf. models S1A1 and S1B1 above) for completeness.

\section{Variables included in the analysis datasets}

Additional file 2: Table S2 is a comprehensive list of the variables used in the analyses. The studies will generally contain only a subset of the complete list and therefore each dataset will participate in only a subset of the proposed analyses. In addition to the variables contained in the datasets, we will gather additional information about the datasets to allow us to evaluate particular sources of heterogeneity through meta-analyses of refined subsets of the datasets.

Variables gathered about each dataset:

1. Genetic ancestry (datasets are stratified by ancestry).

2. Cross-sectional or longitudinal.
3. Information gathered via interview or questionnaire.

4. Information on stress and depression is based on current or lifetime reports.

\section{Additional secondary analyses}

As indicated above, a variety of secondary, interpretive analyses are planned. In addition to the alternate genotype codings and covariates listed above, tests of heterogeneity between datasets are expected to lead to secondary meta-analyses of results from more homogeneous datasets. In particular, it has been argued that many studies that have failed to replicate the original studies lacked in-depth environmental measures [30]. We will investigate potential differences in results between brief, self-report questionnaire measures of stressful life events and face-to-face interviews as well as differences due to the type of study and the diagnostic system used for assessment. Therefore, in addition to subsets identified through tests for heterogeneity, stratified meta-analyses based on the following factors are planned:

1. Genetic Ancestry (e.g. European, Asian, Pacific Island, Admixed European-African).

2. Study Type (e.g. cross-sectional versus longitudinal data collection).

3. Assessment type (e.g. interview versus questionnaire, diagnostic system).

4. Stress and depression phenotypes are based on current state or lifetime reports.

One source of heterogeneity within an individual study is the source of stress. A subset of our participating groups minimized this particular source of heterogeneity by ascertaining subjects that had all been exposed to a uniform stressor (e.g. pregnancy, military conscription, medical residency, coronary heart disease). Because the primary hypothesis supposes that the effect of genotype can be seen most strongly (or only) in subjects exposed to stress, analyses including only these datasets may have particular power to detect the hypothesized effect.

\section{Statistical software}

All analyses will be conducted using $\mathrm{R}$ due to its computational flexibility and free availability.

\section{Group collaboration}

The leadership team is responsible for the project's management decisions and the daily management of this collaboration. A series of conference calls with the contributing investigative teams discussed and reviewed many issues in the design of the meta-analysis. The leadership team, in collaboration with individual study sites, developed the initial protocol based on these discussions. The 
coordinating team at Washington University developed the analysis scripts and will perform the meta-analysis. External beta testers for the scripts were essential for the production and distribution of high-quality scripts to the consortium members.

\section{Ethics}

All participating research groups have appropriate ethics approval for the use of their data in the proposed secondary analyses. A listing of the participating studies, the names of the study representatives collaborating on this project, and the organizations providing ethical review for the studies can be found in Additional file 3: Table S3. The Human Research Protection Office of Washington University in St. Louis has stated that this collaborative meta-analysis paradigm does not require ethical approval beyond what has been provided to the individual participating groups.

\section{Discussion}

\section{Limitations}

In this study, our emphasis is on harmonizing the analysis to maximize the number of studies to be included. The hope is that a large sample size will overcome limitations due to heterogeneity in measurements of depression and environment. This assumption is supported by many GWAS meta-analyses and studies which find many more genetic associations even when compromising on phenotyping, e.g. by considering anyone with a clozapine prescription a case, rather than a formal schizophrenia diagnosis (e.g. $[44,45])$. However, there are limitations to this approach. Although the analysis will be uniform, neither the phenotype nor the environment was measured in a consistent fashion in all studies.

Second, the diathesis-stress model would predict that an alternative approach might be to study a population that was uniformly exposed to a specific stressor. A minority of the studies in our consortium are of this type (e.g. ASPIS (military conscription), Heart and Soul (coronary heart disease), Intern Health Study (medical internship), POUCH (pregnancy)).

Third, limiting the sample size to 300 was done for practical reasons. On the other hand, the small published studies were in their majority those with positive findings [21]). Small samples may be more likely subject to publication bias [23], but also tend to be assessed more carefully, and hence this criterion may exclude some of the best characterized samples.

Fourth, although we will perform a number of specific heterogeneity tests, it is impossible to test all combinations.

Hence, although we will include many possible confounds and expect to have power to detect a robust effect of genotype, a negative finding does not exclude the possibility that a genetic effect exists that is very sensitive to timing or to the method of measuring depression or stress.

\section{Summary}

There is an ongoing debate about what role, if any, 5HTTLPR variation plays in depression. Various studies have argued for (e.g., [15]) or against (e.g., [46]) the proposition that an interaction between 5-HTTLPR variation and stressful life events alters the subsequent risk of depression. Both individual studies and meta-analyses of published results have come to differing conclusions. Numerous issues may contribute to the conflicting results including: heterogeneity among studies (varying study populations, varying definitions of depression, varying measures of stress, prospective versus retrospective assessments, varying analytical models); issues regarding timing of stress and depression; underpowered samples; and publication bias. To address the numerous complexities of the topic of the potential impact of 5-HTTLPR variation on depression, hypothesized as a moderator of the response to stress, we have designed a coordinated meta-analysis that performs consistent, de novo analyses of all available primary data, utilizing variables that are harmonized across the datasets and with analyses that were determined a priori. These analyses will be implemented in a set of primary analyses, arising from the original report of a GxE interaction involving 5-HTTLPR variation, and supplemented by secondary analyses intended to aid in the interpretation of the main findings and to address numerous issues related to possible heterogeneity between the contributing studies. The risk of data mining and of type 1 errors in large, multidimensional datasets such as ours is great, and there is a clear need for consensus on best practice before analyses are conducted. It is our hope that by publishing this protocol in advance of all analyses being conducted, we will minimize potential biases.

\section{Nomenclature}

- 5-HTT: Serotonin transporter gene.

- Official name: SLC6A4, location: 17q11.2. Also known as HTT; 5HTT; OCD1; SERT; 5-HTT; SERT1; hSERT.

- Summary: This gene encodes an integral membrane protein that transports the neurotransmitter serotonin from synaptic spaces into presynaptic neurons. The encoded protein terminates the action of serotonin and recycles it in a sodium-dependent manner. This protein is a target of psychomotor stimulants, such as amphetamines and cocaine, and is a member of the sodium:neurotransmitter symporter family.

- 5-HTTLPR: serotonin-transporter-linked polymorphic region. Summary: A repeat length 
polymorphism in the promoter of the 5-HTT gene that has been shown to affect the rate of serotonin uptake. The short ("s") allele is associated with lower transcriptional efficiency of the promoter compared to the long ("l") allele.

- GxE: Gene-environment interaction.

\section{Additional files}

\author{
Additional file 1: Table S1. Invited groups that are not currently \\ participating.
}

Additional file 2: Table S2. Variables used for the analyses.

Additional file 3: Table S3. Human Research Protection Review for participating studies.

\section{Competing interests}

The authors have no competing interests to declare.

\section{Authors' contributions}

LJB and NB initially conceived of the study. RCC recruited the participating studies, led the protocol design discussions, and drafted the protocol. All authors contributed to the design of the protocol. LB and RCC drafted the manuscript. All authors helped edit the manuscript and approved the final manuscript.

\section{Acknowledgements}

This manuscript was written on behalf of, and in consultation with, the 5HTTLPR, Stress, and Depression Consortium, which consists of the following studies:

Avon Longitudinal Study of Parents and Children (ALSPAC), Athens Study of Psychosis Proneness and Incidence of Schizophrenia (ASPIS), University Mental Health Research Institute, Athens, Greece, Australian Temperament Project (ATP), BIGSIBS, Christchurch Health and Development Study (CHDS), Chinese Academy of Medical Sciences. Cognitive Function and Mood Study (CoFaMS), University of Adelaide, Collaborative Study on the Genetics of Alcoholism (COGA), Collaborative Genetic Study of Nicotine Dependence (COGEND), Community Twin and Longitudinal Twin Samples and National Youth and Family Study (CTS-LTS and NYFS), Depression Case-control study (DeCC), European Prospective Investigation into Cancer - Norfolk (EPICNorfolk), Enquête de Santé Psychologique - Risques, Incidence et Traitement Project (ESPRIT), The Genesis 12-19 Study (G1219), Genetic Study of Bipolar Disorder, GENESIS: GEnetics, NEuropsychology, functional neurolmaging of Suicidal behavior (Genetics Study of Suicidal Behaviour), Grady Trauma Project (GTP), Heart and Soul Study, Intern Health Study, Mannheim Study of Children at Risk (MARS), Michigan Longitudinal Study (MLS), MoodInflame (Münster), Universität Münster, Münster Neuroimaging Study, Universität Münster, Netherlands Study on Depression and Anxiety (NESDA), New molecules in mood disorders: a genomic, neurobiological and systems approach in animal models and human disorder (NEWMOOD), Netherlands Twin Register (Adult NTR and Young NTR), Personality And Total Health through life (PATH), Pregnancy Outcomes and Community Health Studies) based 5-HTTLPR gene-environment interaction for meta-analysis (POUCH), CIBERSAM University of Granada, Spain (PREDICT-Gene), The Queensland Institute of Medical Research Twin and Family Study (QIMR Twin), Centre for Clinical Research, Västerås, Sweden. Survey of Adolescent Life in Vestmanland 2001 and 2006 (SALVe 2001 and SALVe 2006), Social Environment and Biomarkers of Aging Study (SEBAS), Study of Health in Pomerania (SHIP), TRacking Adolescents' Individual Lives Survey (TRAILS), University of Bologna, Institute of Psychiatry, Department of Biomedical and Neuromotor Sciences, Bologna, Italy, University of Molise, Department of Health Sciences, Campobasso, Italy, and Victoria Adolescent Health Care Study (VAHCS). The authors would like to acknowledge the following individuals, for their contributions to the development of the protocol and their roles in the creation of the participating studies:

Kaarin J. Anstey, Ricardo Araya, Volker Arolt, Cecilia Åslund, Gyorgy Bagdy, Tobias Banaschewski, Bernhard T. Baune, Dorret Boomsma, Daniel Brandeis, Peter Butterworth, Vladimir Carli, Jorge Cervilla, Helen Christensen, Sarah
Cohen-Woods, Erika Comasco, Philippe Courtet, William L. Coventry, Udo Dannlowski, Eco de Geus, Bill Deakin, Katharina Domschke, Harmen Draisma, Simon Easteal, Thalia Eley, Anne Farmer, Helen L. Fisher, lan Gotlib, Hans Jörgen Grabe, Blanca Gutiérrez, Brett Haberstick, John Hewitt, Amy Horton, John Horwood, Jouke-Jan Hottenga, Isabelle Jaussent, Anthony Jorm, Gabriella Juhasz, Manfred Laucht, Judit Lazary, Jerzy Leppert, Kathryn Lester, Keriann Little, Alain Malafosse, Laura Mandelli, Nicholas G. Martin, Peter McGuffin, Christel Middeldorp, Camelia Minica, Grant W. Montgomery, Matthias Nauck, Esther Nederhof, Kent W. Nilsson, Niklas Nordquist, Albertine J. Oldehinkel, Emilie Olié, Lars Oreland, Johan Ormel, Christian Otte, Brenda Penninx, Wouter Peyrot, Karen Ritchie, Bryan Rodgers, Marco Sarchiapone, Andrea Schulz, Christian Schwahn, Srijan Sen, Alessandro Serretti, Grant Sinnamon, Rickard L Sjöberg, Johannes Smit, Nicholas Stefanis, Paul Surtees, Sandra Villafuerte, Henry Völzke, Nick Wainwright, Mary Whooley, Gonneke Willemsen, Naomi R. Wray, and Robert A Zucker.

This work was supported by National Institutes of Health grants R21 DA033827 and R01 DA026911 from the National Institute on Drug Abuse, P01 CA089392 from the National Cancer Institute, and U10 AA008401 from the National Institute on Alcohol Abuse and Alcoholism.

\section{Author details}

${ }^{1}$ Department of Medicine, Washington University School of Medicine, St. Louis, MO, USA. 'Division of Biostatistics, Washington University School of Medicine, St. Louis, MO, USA. ${ }^{3}$ Department of Social Policy and Intervention, University of Oxford, Oxford, UK. ${ }^{4}$ Department of Epidemiology and Biostatistics, Michigan State University, East Lansing MI, USA. ${ }^{5}$ nstitute of Psychiatric Research, Departments of Psychiatry and Medical and Molecular Genetics, Indiana University School of Medicine, Indianapolis, IN, USA. ${ }^{6}$ Molecular and Behavioral Neuroscience Institute, University of Michigan, Ann Arbor, MI, USA. ${ }^{7}$ Department of Human Genetics, University of Michigan, Ann Arbor MI, USA. ${ }^{8}$ Department of Psychiatry, University of Michigan, Ann Arbor MI, USA. ${ }^{9}$ Christchurch Health and Development Study, Department of Psychological Medicine, University of Otago, Christchurch, New Zealand. ${ }^{10} \mathrm{MRC}$ Integrative Epidemiology Unit, UK Centre for Tobacco Control Studies and School of Experimental Psychology, University of Bristol, Bristol, UK. ${ }^{11}$ Department of Genetics, Washington University School of Medicine, St. Louis, MO, USA. ${ }^{12}$ Department of Psychiatry, Washington University School of Medicine, Saint Louis, MO, USA.

Received: 4 October 2013 Accepted: 14 October 2013

Published: 12 November 2013

\section{References}

1. Sullivan PF, Neale MC, Kendler KS: Genetic epidemiology of major depression: review and meta-analysis. Am J Psychiatry 2000, 157:1552-1562.

2. Bierut LJ, Heath AC, Bucholz KK, Dinwiddie SH, Madden PA, et al: Major depressive disorder in a community-based twin sample: are there different genetic and environmental contributions for men and women? Arch Gen Psychiatry 1999, 56:557-563.

3. Kendler KS, Gardner CO, Prescott CA: Are there sex differences in the reliability of a lifetime history of major depression and its predictors? Psychol Med 2001, 31:617-625.

4. Kendler KS, Neale MC, Kessler RC, Heath AC, Eaves LJ: The lifetime history of major depression in women. Reliability of diagnosis and heritability. Arch Gen Psychiatry 1993, 50:863-870.

5. McGuffin P, Katz R, Rutherford J: Nature, nurture and depression: a twin study. Psychol Med 1991, 21:329-335.

6. McGuffin P, Katz R, Watkins S, Rutherford J: A hospital-based twin register of the heritability of DSM-IV unipolar depression. Arch Gen Psychiatry 1996, 53:129-136.

7. Ripke S, Wray NR, Lewis CM, Hamilton SP, Weissman MM, et al: A megaanalysis of genome-wide association studies for major depressive disorder. Mol Psychiatry 2013, 18:497-511.

8. Cohen-Woods S, Craig IW, McGuffin P: The current state of play on the molecular genetics of depression. Psychol Med 2013, 43:673-687.

9. Kendler KS, Kessler RC, Walters EE, MacLean C, Neale MC, et al: Stressful life events, genetic liability, and onset of an episode of major depression in women. Am J Psychiatry 1995, 152:833-842.

10. Lesch KP, Greenberg MD, Higley JD, Bennett A, Murphy DL: Serotonin transporter, personality, and behavior: toward a disection of gene-gene 
and gene-environment interaction. In Molecular genetics and the human personality. Edited by Benjamin J, Ebstein RP, Belmaker RH. Washington, DC: American Psychiatric Association (APA); 2002: pp. 109-136.

11. Lesch KP, Bengel D, Heils A, Sabol SZ, Greenberg BD, et al: Association of anxiety-related traits with a polymorphism in the serotonin transporter gene regulatory region. Science 1996, 274:1527-1531.

12. Akey J, Jin L, Xiong M: Haplotypes vs single marker linkage disequilibrium tests: what do we gain? Eur J Hum Genet 2001, 9:291-300.

13. Nakamura M, Ueno S, Sano A, Tanabe H: The human serotonin transporter gene linked polymorphism (5-HTTLPR) shows ten novel allelic variants. Mol Psychiatry 2000, 5:32-38.

14. Araya R, Hu X, Heron J, Enoch MA, Evans J, et al: Effects of stressful life events, maternal depression and 5-HTTLPR genotype on emotional symptoms in pre-adolescent children. Am J Med Genet B Neuropsychiatr Genet 2009, 150B:670-682.

15. Caspi A, Sugden K, Moffitt TE, Taylor A, Craig IW, et al: Influence of life stress on depression: moderation by a polymorphism in the $5-\mathrm{HTT}$ gene. Science 2003, 301:386-389.

16. Munafo MR, Durrant C, Lewis G, Flint J: Gene X environment interactions at the serotonin transporter locus. Biol Psychiatry 2009, 65:211-219.

17. Belsky J, Pluess M: Beyond diathesis stress: differential susceptibility to environmental influences. Psychol Bull 2009, 135:885-908.

18. Uher R, McGuffin P: The moderation by the serotonin transporter gene of environmental adversity in the aetiology of mental illness: review and methodological analysis. Mol Psychiatry 2008, 13:131-146.

19. Brown GW, Harris TO: Depression and the serotonin transporter 5-HTTLPR polymorphism: a review and a hypothesis concerning gene-environment interaction. J Affect Disord 2008, 111:1-12.

20. Clarke H, Flint J, Attwood AS, Munafo MR: Association of the 5- HTTLPR genotype and unipolar depression: a meta-analysis. Psychol Med 2010, 40:1767-1778

21. Karg K, Burmeister M, Shedden $K$, Sen S: The serotonin transporter promoter variant (5-HTTLPR), stress, and depression meta-analysis revisited: evidence of genetic moderation. Arch Gen Psychiatry 2011, 68:444-454

22. Risch $N$, Herrell $R$, Lehner $T$, Liang $K Y$, Eaves $L$, et al: Interaction between the serotonin transporter gene (5-HTTLPR), stressful life events, and risk of depression: a meta-analysis. JAMA 2009, 301:2462-2471.

23. Duncan LE, Keller MC: A critical review of the first 10 years of candidate gene-by-environment interaction research in psychiatry. Am J Psychiatry 2011, 168:1041-1049.

24. Munafo MR, Flint J: Replication and heterogeneity in gene $x$ environment interaction studies. Int J Neuropsychopharmacol 2009, 12:727-729.

25. Uher R, Caspi A, Houts R, Sugden K, Williams B, et al: Serotonin transporter gene moderates childhood maltreatment's effects on persistent but not single-episode depression: replications and implications for resolving inconsistent results. J Affect Disord 2011, 135:56-65.

26. Button KS, loannidis JP, Mokrysz C, Nosek BA, Flint J, et al: Power failure: why small sample size undermines the reliability of neuroscience. Nat Rev Neurosci 2013, 14:365-376.

27. Kim JM, Stewart R, Kim SW, Yang SJ, Shin IS, et al: Interactions between life stressors and susceptibility genes (5-HTTLPR and BDNF) on depression in korean elders. Biol Psychiatry 2007, 62:423-428.

28. Covault J, Tennen H, Armeli S, Conner TS, Herman Al, et al: Interactive effects of the serotonin transporter 5-HTTLPR polymorphism and stressful life events on college student drinking and drug use. Biol Psychiatry 2007, 61:609-616

29. Wilhelm K, Mitchell PB, Niven H, Finch A, Wedgwood L, et al: Life events, first depression onset and the serotonin transporter gene. $\mathrm{Br} J$ Psychiatry 2006, 188:210-215

30. Caspi A, Hariri AR, Holmes A, Uher R, Moffitt TE: Genetic sensitivity to the environment: the case of the serotonin transporter gene and its implications for studying complex diseases and traits. Am J Psychiatry 2010, 167:509-527.

31. Taylor SE, Way BM, Welch WT, Hilmert CJ, Lehman BJ, et al: Early family environment, current adversity, the serotonin transporter promoter polymorphism, and depressive symptomatology. Biol Psychiatry 2006, 60:671-676.

32. Chipman $P$, Jorm AF, Prior M, Sanson A, Smart D, et al: No interaction between the serotonin transporter polymorphism (5-HTTLPR) and childhood adversity or recent stressful life events on symptoms of depression: results from two community surveys. Am J Med Genet $B$ Neuropsychiatr Genet 2007, 144B:561-565.

33. Chipman $P$, Jorm AF, Tan $X Y$, Easteal $S$ : No association between the serotonin-1A receptor gene single nucleotide polymorphism rs6295C/G and symptoms of anxiety or depression, and no interaction between the polymorphism and environmental stressors of childhood anxiety or recent stressful life events on anxiety or depression. Psychiatr Genet 2010, 20:8-13.

34. Kaufman J, Yang BZ, Douglas-Palumberi H, Grasso D, Lipschitz D, et al: Brain-derived neurotrophic factor-5-HTTLPR gene interactions and environmental modifiers of depression in children. Biol Psychiatry 2006, 59:673-680

35. Kaufman J, Yang BZ, Douglas-Palumberi H, Houshyar S, Lipschitz D, et al: Social supports and serotonin transporter gene moderate depression in maltreated children. Proc Natl Acad Sci USA 2004, 101:17316-17321.

36. Zammit S, Lewis G, Dalman C, Allebeck P: Examining interactions between risk factors for psychosis. Br J Psychiatry 2010, 197:207-211.

37. Chen LS, Saccone NL, Culverhouse RC, Bracci PM, Chen CH, et al: Smoking and genetic risk variation across populations of European, Asian, and African American ancestry-a meta-analysis of chromosome 15q25. Genet Epidemiol 2012, 36:340-351

38. Hartz SM, Short SE, Saccone NL, Culverhouse R, Chen L, et al: Increased genetic vulnerability to smoking at CHRNA5 in early-onset smokers. Arch Gen Psychiatry 2012, 69:854-860.

39. Saccone NL, Culverhouse RC, Schwantes-An TH, Cannon DS, Chen X, et al: Multiple independent loci at chromosome 15q25.1 Affect smoking quantity: a meta-analysis and comparison with lung cancer and COPD. PLoS Genet 2010, 6

40. Sniderman AD, Williams K, Contois JH, Monroe HM, McQueen MJ, et al: A meta-analysis of low-density lipoprotein cholesterol, non-high-density lipoprotein cholesterol, and apolipoprotein B as markers of cardiovascular risk. Circ Cardiovasc Qual Outcomes 2011, 4:337-345.

41. Bernstein DP, Fink L, Handelsman L, Foote J, Lovejoy $M$, et al: Initial reliability and validity of a new retrospective measure of child abuse and neglect. Am J Psychiatry 1994, 151:1132-1136.

42. Brugha TS, Cragg D: The list of threatening experiences: the reliability and validity of a brief life events questionnaire. Acta Psychiatr Scand 1990, 82:77-81.

43. Robins L, Cottler L, Bucholz K, Compton W: Diagnostic interview schedule for DSM-IV, pp. St. Louis (MO): Washington University School of Medicine; 1995.

44. Hamshere ML, Walters JT, Smith R, Richards AL, Green E, Grozeva D, Jones I, Forty L, Jones L, Gordon-Smith K, et al: Genome-wide significant associations in schizophrenia to ITIH3/4, CACNA1C and SDCCAG8, and extensive replication of associations reported by the Schizophrenia PGC. Mol Psychiatry 2013, 18(6):708-712.

45. Ripke S, Hubner T, Mennigen E, Muller KU, Rodehacke S, et al: Reward processing and intertemporal decision making in adults and adolescents: the role of impulsivity and decision consistency. Brain Res 2012, 1478:36-47

46. Fergusson DM, Horwood LJ, Miller AL, Kennedy MA: Life stress, 5-HTTLPR and mental disorder: findings from a 30-year longitudinal study. Br J Psychiatry 2011, 198:129-135.

\section{doi:10.1186/1471-244X-13-304}

Cite this article as: Culverhouse et al.: Protocol for a collaborative metaanalysis of 5-HTTLPR, stress, and depression. BMC Psychiatry 2013 13:304. 